\title{
A Unified Line-of-Sight Probability Model for Commercial 5G Mobile Network Deployments
}

\author{
Dave Townend, Stuart D. Walker, Adrian Sharples and Andy Sutton
}

\begin{abstract}
In this paper we present a novel analytical approach used to derive a new multi-scenario line-of-sight (LOS) probability model for cellular network deployments in the UK. The approach considers the use of lamp post databases as statistically representative geo-spatial data points for evaluation of LOS likelihood from macro cellular base stations. Crucially, the proposed model is built on a high resolution $(0.25-1 \mathrm{~m})$ 3D digital surface model underpinned by real network and environmental datasets and validated with supporting field measurements. This work unifies all common cell site classification types; urban, suburban and rural into a single 3D LOS statistical probability model whilst also addressing the influence of endpoint height properties up to $10 \mathrm{~m}$. The contributions outlined in this paper have applications in statistical path loss modelling and coverage/outage probability. They also have direct application in deployment modelling of mmWave (millimeter wave) mobile access networks (24.25-52.6 GHz) and wireless $x$-haul transport networks $(71-174.8 \mathrm{GHz})$.

Index Terms-mobile network, deployment modelling, millimeter wave (mmWave), channel modelling, line-of-sight probability
\end{abstract}

\section{INTRODUCTION}

The relentless capacity demand on cellular networks is the fundamental factor driving spectrum usage into ever higher bands. This has been no more evident than in the adoption of mmWave spectrum (typically considered to be bands above $24 \mathrm{GHz}$ ) in 3GPP standards as of release 15 [1]. Whilst progression towards mmWave radio promises a step change in capacity resulting from larger channel bandwidth availability, it equally presents significant deployment challenges relative to traditional access bands below $6 \mathrm{GHz}$ [2]. A high path loss exponent ( $n \geq 2.55$ [3]), susceptibility to blockage and unfavourable diffraction properties as well as poor in-building penetration characteristics [4] present coverage constraints for conventional outdoor environments.

Accurate deployment modelling and reliable system level assessments in built up environments remain the same regardless of how mmWave spectrum is ultimately utilised. Although the non line-of-sight (NLOS) or near line-of-sight (nLOS) propagation characteristics of high frequency radio systems have been experimentally proven in real environments [5] [6] [4], the accuracy of corresponding theoretical predictions is not well reported particularly at scale. It is often the resolution of the radio planning or simulation environment relative to the frequencies of interest $(\lambda=0.012 \mathrm{~m}$ for lowest end 3GPP FR2 band) that is the fundamental barrier to reliable exploitation of NLOS propagation paths in commercial deployments. Collection of environmental data sufficient to more accurately predict mmWave diffraction, scattering and reflection characteristics is generally considered cost prohibitive and as such the desired service availability targets underpinning new technology investments may only be achieved in the LOS case. In its fundamental form, the LOS evaluation can be considered frequency independent (optical), however the underlying practical validity of any LOS assessment should be considered as a function of frequency owing to the necessary Fresnel zone clearance.

In this work we consider two direct applications of mmWave use on macro base station sites abstracted by the LOS probability model to assess deployment viability. In the first case, we consider relevance to the transport network (TN) and in the second, to the radio access network (RAN). In the TN case, we examine the role of macro sites as backhaul (evolving to fronthaul) wireless transmission hubs for future dense network architectures. This includes the use of integrated access and backhaul (IAB) nodes [7] as well as disaggregated RAN [8] and cell-free deployment models [9]. Here, the LOS probability for new street level infrastructure locations (lamp posts) represents the opportunity of using mmWave transmission links for backhaul to new cooperative radio nodes close to the end user at street level. For the RAN case, we assume the underlying technology to be mobile access at bands around 26 $\mathrm{GHz}$ where the LOS probability represents an approximation of coverage availability. For such applications, the probabilistic assessment is for LOS validity to slow moving or static user equipment (UEs) at heights of $\sim 1.5 \mathrm{~m}$ for pedestrians on the pavement and $\sim 5 \mathrm{~m}$ for fixed wireless access (FWA) terminals affixed to roadside buildings.

To address these two areas, we present two new contributions evaluating the 3D LOS probability channel in a largescale commercial network. Section II outlines the simulation approach used to derive the LOS probability results and associated statistical data sets. Section III outlines the transport network use case detailing results for LOS between real lamp posts and macro cell masts in urban, suburban and rural cell types. These results are evaluated against recognised and published probability models. Section IV addresses the generalised height dependent LOS probability relevant to radio access network use cases. Here, the impact of endpoint height variation on the same data points is accessed allowing a new model definition to be proposed in this paper. Finally, Section $\mathrm{V}$ summarises this work and direction of associated research.

\section{A. Line-of-Sight Probability Models}

Development of statistical channels models such as the LOS probability model has arisen through the need to treat the path loss exponents of LOS and NLOS cases differently. The 
TABLE I: Macro Cellular Line-of-Sight Probability Models.

\begin{tabular}{|c|c|c|}
\hline Model & Definition & Parameters \\
\hline $\begin{array}{l}3 \mathrm{GPP} \\
(\mathrm{UMa}) \\
{[10]}\end{array}$ & 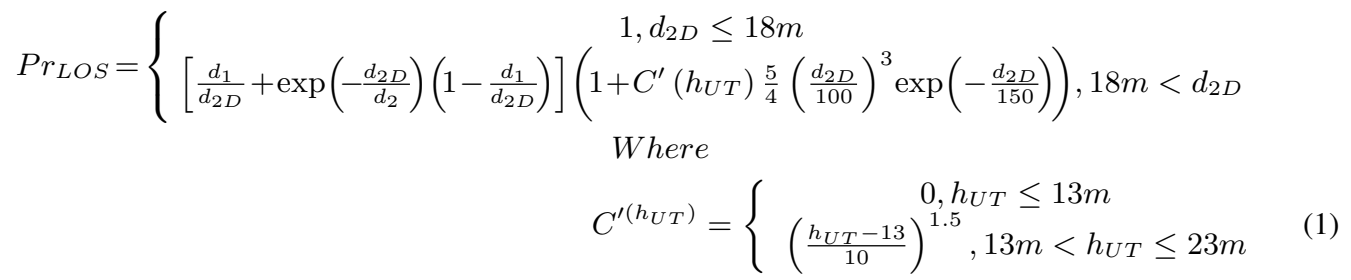 & $\begin{array}{l}d_{1}=18 \\
d_{2}=63\end{array}$ \\
\hline $\begin{array}{l}d_{1} / d_{2} \\
(\mathrm{UMa}) \\
{[11]}\end{array}$ & $\operatorname{Pr}_{L O S}=\left(\min \left(\frac{d_{1}}{d_{2 D}}, 1\right)\left(1-\exp \left(-\frac{d_{2 D}}{d_{2}}\right)\right)+\exp \left(-\frac{d_{2 D}}{d_{2}}\right)\left(1+C^{\prime}\left(h_{U T}\right)\right)\right)$ & $\begin{array}{l}d_{1}=20 \\
d_{2}=66\end{array}$ \\
\hline $\begin{array}{c}\text { NYU } \\
\text { Squared } \\
\text { (UMa) } \\
{[12]}\end{array}$ & $\operatorname{Pr}_{L O S}=\left(\left(\min \left(\frac{d_{1}}{d_{2 D}}, 1\right)\left(1-\exp \left(-\frac{d_{2 D}}{d_{2}}\right)\right)+\exp \left(-\frac{d_{2 D}}{d_{2}}\right)\left(1+C^{\prime}\left(h_{U T}\right)\right)\right)\right)^{2}$ & $\begin{array}{c}d_{1}=20 \\
d_{2}=160\end{array}$ \\
\hline $\begin{array}{l}3 \mathrm{GPP} \\
(\mathrm{RMa}) \\
{[10]}\end{array}$ & $\operatorname{Pr}_{L O S}=\left\{\begin{array}{c}1, d_{2 D} \leq 10 m \\
\exp \left(-\frac{d_{2 D}-10}{1000}\right), 10 m<d_{2 D}\end{array}\right.$ & None \\
\hline
\end{tabular}

LOS probability model describes the likelihood $\operatorname{Pr}_{L O S}$ of an endpoint being in clear LOS of the base station as a function of the two dimensional distance $d_{2 D}$ (in metres) between them.

In recent years, most focus has been on the urban macro (UMa) LOS scenario. The UMa LOS probability model was first adopted as part of the 3D channel model definition in 3GPP release 12 based on ITU and WINNER II definitions [13][14]. These definitions did not originally account for the influence of the endpoint height $h_{U T}$ (in metres) which was subsequently introduced in the form of (1) in 3GPP TR 36.873 [15] and later 3GPP TR 38.900/38.901 [10]. These revisions were derived using ray-tracing techniques of a simulated urban environment underpinned by assumptions about typical building and base station properties [16]. Significant effort has been applied to experimental verification and optimisation of the UMa case in recent years primarily resulting from a focused effort on feasibility of mmWave 5G mobile access where coverage characteristics are principally considered LOS dependent. Further revisions to the parametrisations of the UMa model, the ' $d_{1} / d_{2}$ ' model in (2) have subsequently been proposed [11] including the addition of a squared term modifier for increased resolution [12], the New York University 'NYU squared' model in (3). These proposals have been derived primarily from map based analysis or measurement campaigns of dense urban Manhattan grid style environments.

Conspicuous by its absence is a recognised LOS model for the suburban macro (SMa) scenario which typically accounts for a higher proportion of cells in a European network [17]. The suburban case has a basic definition defined in the WINNER II channel model [14] although this was not brought forward into ITU recommendations or 3GPP guidelines.

While a dedicated rural macro (RMa) model is defined in 3GPP there has been little development or experimental characterisation of the rural case since its adoption as part of the wider 3D channel model into 3GPP guidelines. The RMa LOS model was again adopted from historical ITU and WINNER empirical models based on relatively limited data sets and specific assumptions and approximations regarding the location of obstacles in the direct path. The result is the relatively simple definition in (4) which offers no fitting parameters or variables to account for endpoint height profile. These notable and recognised LOS probability models are outlined in Table I and assessed as part of the environmental simulation analysis in Section III.

\section{Simulation EnVironment}

A deterministic simulation approach was developed to quantify the LOS properties of a real mobile network deployment. Here, a highly detailed 3D environmental model was built for large areas of the UK covering a number of representative large and mid-size towns and cities including central London and Manchester and covering a total area of approximately $1875 \mathrm{sq} \mathrm{km}$. The environmental model was constructed of multi-resolution LIDAR surveys publicly available from the Department for Environmental and Rural Affairs [18] with resolutions $0.25 \mathrm{~m}, 0.5 \mathrm{~m}$ and $1 \mathrm{~m}$. These surveys were resampled into a single digital surface model (DSM) of raster resolution $0.25 \mathrm{~m}$ using MapInfo GIS software. The resulting worst-case error margin exists in areas covered only by the $1 \mathrm{~m}$ resolution LIDAR survey and assumed to be no more 


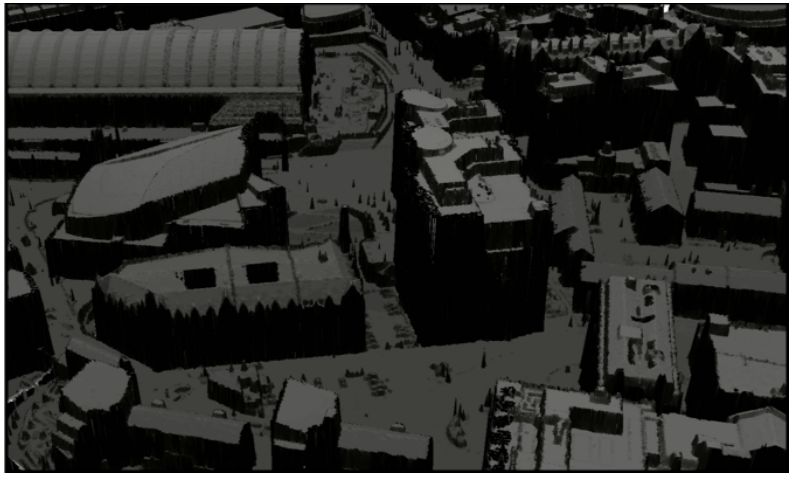

Fig. 1: DSM 3D graphical representation of urban Manchester.

than $+/-0.5 \mathrm{~m}$, this accounts for $52 \%$ of the model area. Notable 3D projections in the DSM were characterised through augmented terrain, building, road and green space datasets. The DSM was then complemented with 3D mobile network operator infrastructure sites (BT/EE) and local authority street lighting and lamp post sites. The DSM simulation environment comprises of 2154 macro base station sites which account for a little over $10 \%$ of the total national network. Prior to simulations being conducted, the cell size classification definitions were derived for alignment with conventional ITU and 3GPP definitions of urban, suburban and rural macro cells. Voronoi polygons were constructed from site locations and the 2D inter site distance (ISD) between each geometric neighbour calculated through construction of the associated Delaunay triangulation lines. The Voronoi coverage definition allows abstraction of the network topology independent of specific cell site configurations which may influence the link layer coverage areas such as operating frequency, radio access technology, transmit power level and antenna gain and orientation. Cells with mean ISD $\leq 500 \mathrm{~m}$ were classified and analysed as UMa, $>500 \mathrm{~m}$ and $\leq 1299 \mathrm{~m}$ as SMa and $>1299 \mathrm{~m}$ and $\leq 1732 \mathrm{~m}$ as $\mathrm{RMa}$, the full distribution of cell sites in a UK network is given in [17]. A basic ray tracing technique was used where vector lines are constructed between each macro site and all lamp posts within its Voronoi coverage area where it is assumed to be the best server. The propagation path between base station height and each corresponding lamp post height was only validated where no pixels were intersected by the underlying surface model (i.e. buildings/terrain) as in Fig. 2.

\section{Street Furniture Endpoints - A Transport NeTWORK USE CASE}

LOS validation was carried out between existing macro sites and over 250,000 lamp posts to evaluate the validity of the published LOS models for a real network. The distribution profiles of lamp post height properties are highlighted in Fig. 3 and the proximity to their serving cell in Fig. 4. The utilisation of lamp post data in this study is key, not only does it provide insight into the viability of wirelessly aggregating backhaul or fronthaul from new low power street cells to macro sites as discussed in Section I, but the lamp posts also

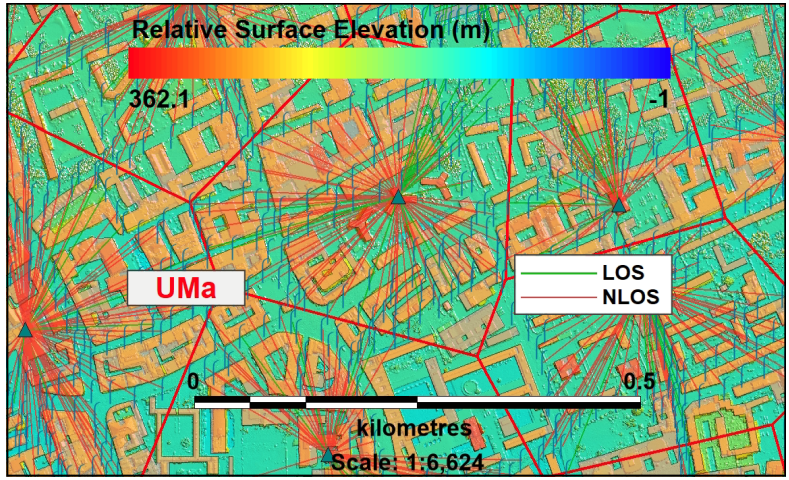

Fig. 2: DSM 2D graphical representation of urban London.

act as a credible proxy for many alternative mmWave use cases. Small variations in the spatial properties of this dataset allow the lamp post locations to be used as an accurate and representative sample point across the coverage environment for evaluation of the radio access network use cases also considered in Section I. For the radio access network case, the endpoints can be considered representative of an outdoor user distribution (although not user density distribution) since these data points are logically equivalent to the flow of outdoor mobile user terminals in a real network. As such, any associated theoretical modelling of the outdoor mobile user scenarios could also be aligned to the distributions describing the proximity of lamp posts to the serving cell in Fig.4 rather than assumed random and uniformly distributed as per ITU and 3GPP simulation guidelines.

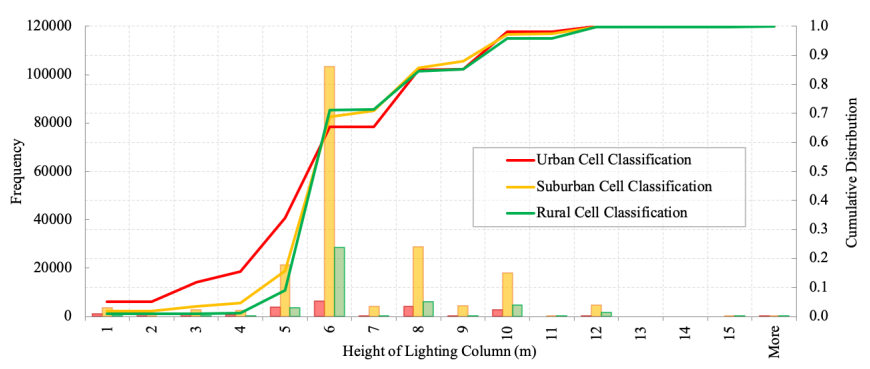

Fig. 3: Lamp post height distribution.

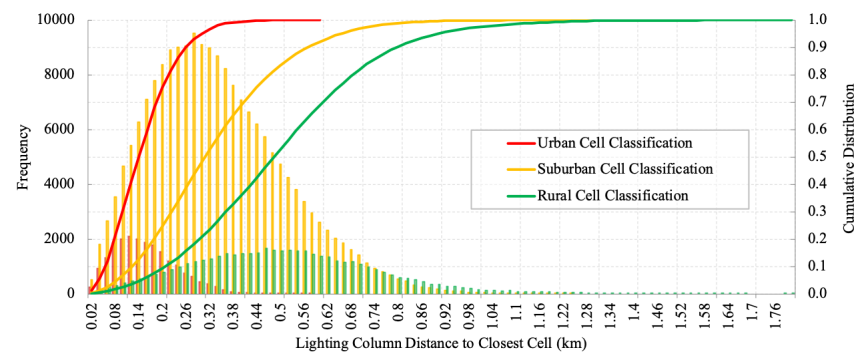

Fig. 4: Lamp post proximity to nearest cell distribution.

Examination of the height distribution of the real lamp posts in Fig. 3 shows that $99.9 \%$ of the data points are below a height of $13 \mathrm{~m}$. This is significant as the existing LOS probability 
models outlined in Table I would have no contribution from the height profile term $C^{\prime\left(h_{U T}\right)}$ for data points below $13 \mathrm{~m}$. As a result, the LOS probability for the entire lamp post dataset, regardless of individual height, would remain solely a function of the distance from the base station.

\section{A. Urban Scenario}

Results for LOS probability across all urban classified cells are outlined in Fig. 5. The published models relevant to the UMa scenario from Table I are also overlaid using both the recommended values for the parameters $d_{1}$ and $d_{2}$, as well as optimised values achieved through minimisation of mean squared error (MSE) to the DSM data which are also summarised in Table II. Optimisation of the existing model fitting parameters result in the same probability distribution for each model where $d_{1}=0$ and $d_{2}=44$, this is to be expected as these models are based on the same fundamental derivation. In order to maintain statistical relevance for distance intervals with a lower number of data points, a uniform data point count sampling approach is used across the $\mathrm{x}$-axis as opposed to uniform distance sampling.

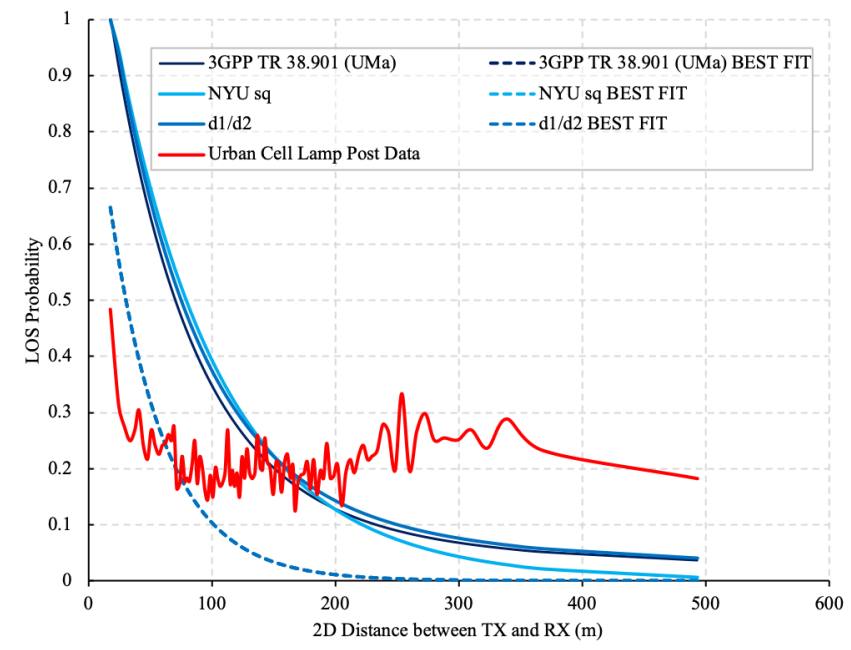

Fig. 5: LOS probability of urban lamp posts.

TABLE II: Urban Macro LOS Probability Models.

\begin{tabular}{|c|c|c|c|}
\hline Model & \multicolumn{2}{|c|}{ Parameters } & MSE \\
\hline \hline 3GPP UMa [10] & Default & $\begin{array}{c}d_{1}=18 \\
d_{2}=63\end{array}$ & 3.88 \\
\cline { 2 - 4 } & Best Fit & $\begin{array}{c}d_{1}=0 \\
d_{2}=44\end{array}$ & 0.54 \\
\hline$d_{1} / d_{2}[11]$ & Default & $\begin{array}{c}d_{1}=20 \\
d_{2}=66\end{array}$ & 4.57 \\
\cline { 2 - 4 } & Best Fit & $\begin{array}{c}d_{1}=0 \\
d_{2}=44\end{array}$ & 0.54 \\
\hline NYU (Squared) [12] & Default & $\begin{array}{c}d_{1}=20 \\
d_{2}=160\end{array}$ & 5.05 \\
\cline { 2 - 4 } & Best Fit & $\begin{array}{c}d_{1}=0 \\
d_{2}=44\end{array}$ & 0.54 \\
\hline
\end{tabular}

In all cases, existing model definitions show poor agreement with the calculated data set which demonstrate a much lower
LOS probability towards the cell centre (within $\sim 150 \mathrm{~m}$ ) with a peak probability of only 0.48 within $20 \mathrm{~m}$. Results also demonstrate a much flatter profile across the remaining cell radius rather than the exponential decay of existing models. On average $22 \%$ of urban lamp posts within the towns and cities analysed could achieve LOS to their serving macro site.

\section{B. Suburban Scenario}

The equivalent LOS probability results for suburban cells are highlighted in Fig. 6 and the tabulated results with parameter fitting detailed in Table III. No formal LOS model for suburban macro cell is recognised in 3GPP or ITU recommendations and so data results have been evaluated against the equivalent urban models in Table I. As with urban results the suburban case demonstrates a poor fit against the published models even after optimisation. This further highlights the need for a formally recognised definition which can better describe the statistical properties of real network deployments. Overall, the suburban environment provided the highest probability of achieving clear light of sight with $34 \%$ of lamp posts successful.

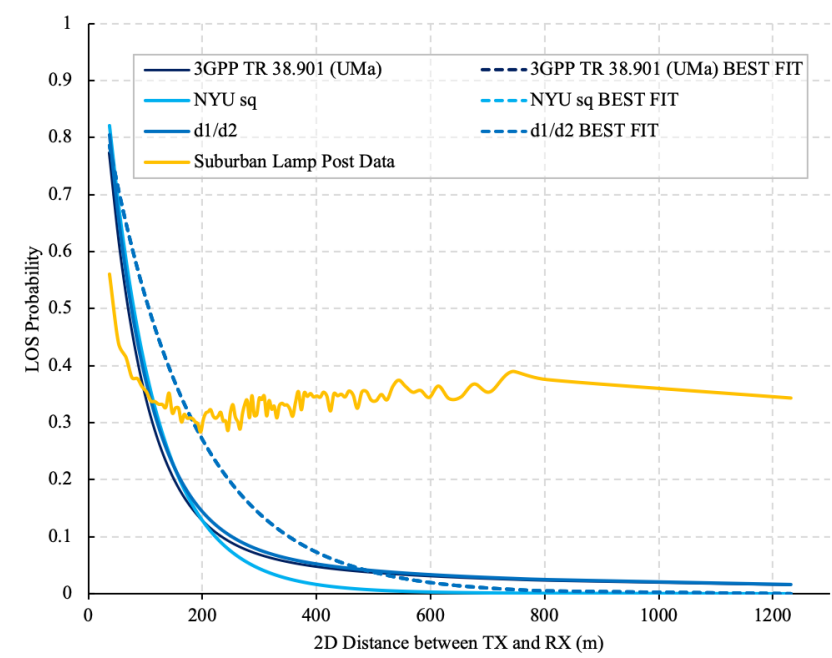

Fig. 6: LOS probability of suburban lamp posts.

TABLE III: Suburban Macro LOS Probability Models.

\begin{tabular}{|c|c|c|c|}
\hline Model & \multicolumn{2}{|c|}{ Parameters } & MSE \\
\hline \hline 3GPP UMa [10] & Default & $\begin{array}{c}d_{1}=18 \\
d_{2}=63\end{array}$ & 1.38 \\
\cline { 2 - 4 } & Best Fit & $\begin{array}{c}d_{1}=0 \\
d_{2}=153\end{array}$ & 0.69 \\
\hline$d_{1} / d_{2}[11]$ & Default & $\begin{array}{c}d_{1}=20 \\
d_{2}=66\end{array}$ & 1.25 \\
\cline { 2 - 4 } & Best Fit & $\begin{array}{c}d_{1}=0 \\
d_{2}=153\end{array}$ & 0.69 \\
\hline NYU (Squared) [12] & Default & $\begin{array}{c}d_{1}=20 \\
d_{2}=160\end{array}$ & 1.54 \\
\cline { 2 - 4 } & Best Fit & $\begin{array}{c}d_{1}=0 \\
d_{2}=305\end{array}$ & 0.69 \\
\hline
\end{tabular}




\section{Rural Scenario}

Rural cell results are shown in Fig. 7 and tabulated in Table IV. There are no optimisation parameters available for curve fitting in the RMa model resulting in a best case MSE of 9.19 and an overall LOS probability of $28 \%$ across the rural dataset.

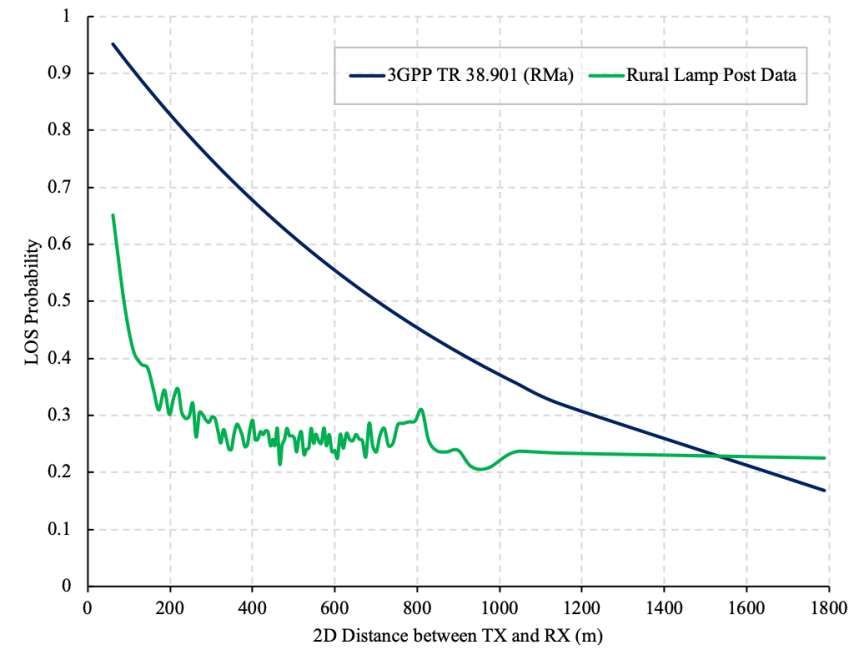

Fig. 7: LOS probability of rural lamp posts.

TABLE IV: Rural Macro LOS Probability Model.

\begin{tabular}{|c|c|c|c|}
\hline Model & \multicolumn{2}{|c|}{ Parameters } & MSE \\
\hline \hline 3GPP RMa [10] & Default & None & 9.19 \\
\hline
\end{tabular}

\section{Experimental Measurement Verification}

The simulated results highlighted in Section III-A - III-C are supported by a measurement campaign aimed at validating LOS prediction accuracy from the DSM environment. A total of 68 measurement points were assessed over two live suburban cell site locations included within the study area during scheduled outage periods. Two measurement methodologies were assessed: a 1-way LOS verification and a 2-way LOS verification. In the 1-way case, the LOS verification is conducted from the base station end with a telephoto camera towards a surveyor with a LOS spotting lamp at the 'terminal' end. In the 2-way case, cameras and spotting lamps were utilised at each end of the link as in Fig. 8. The majority of measurements $66 \%$, were completed with the 1-way setup as this was deemed to be sufficiently accurate based on initial measurements and permitted more time for additional data points to be collected. In both scenarios, measurements are conducted at the localised height of the base station antenna and at $5 \mathrm{~m}$ at the 'terminal' end using a pole mounted camera and LOS spotting lamp. In total, $94 \%$ of the measurement locations distributed around the cell sites agreed with the DSM prediction. In the majority of the incorrectly predicted locations the measurement team cited localised blockages close to either end of the propagation path as the primary factor. Such blockages were likely either blockages below the resolution of the DSM or foliage growth which has occurred between the LIDAR survey date and the
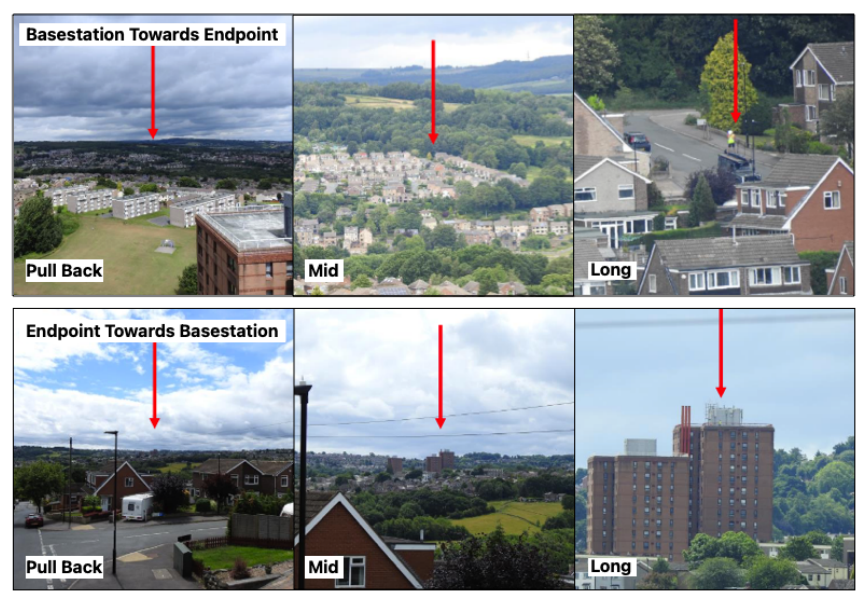

Fig. 8: The 2-way LOS validation measurement.

measurement date (approximately 18 months). In addition, $15 \%$ of the measurement locations were also flagged as subjectively having the potential for Fresnel zone blockage for lower mmWave bands (namely $26 \mathrm{GHz}$ ). While these paths would likely not present an issue at higher fixed service transmission bands foreseen as promising fronthaul connectivity solutions ( $71 \mathrm{GHz}$ 'E-band' to $174 \mathrm{GHz}$ 'D-band') findings do emphasise the suitability of the modelling methodology when considering frequency bands or link distances where the required Fresnel zone clearance approaches the assumed error margin in the deployment model.

\section{Generalised Endpoint Height - A Radio Access Network Use Case}

Here, the generalised LOS case used to determine the influence of the endpoint height is achieved by reconfiguration the same lamp post dataset with modifications to the $3 \mathrm{D}$ height profiles. In the generalised case, all endpoint locations are reconfigured for a consistent height at increments between 1.5 $\mathrm{m}$ and $10 \mathrm{~m}$ above ground. The resulting LOS probability curve for UMa cells is shown in Fig. 9 with the corresponding SMa and RMa scenarios in Fig. 10 and Fig. 11 respectively. For each cell classification, the significance of endpoint height is evident through the spread of results. This further emphasises the need for a model that accurately reflects such factors which are absent in existing models for heights below 13 $\mathrm{m}$. In addition, the absence of a recognised SMa definition and an over simplified RMa definition suggest integration of deployment scenarios in to a common model definition is feasible and could further streamline future refinements. Consequently, a new model is proposed in (5) based on heuristic parameter estimation and minimisation of the mean squared error curve fitting of the DSM results. The resulting predictions are overlaid in Fig. 9-11 with the mean squared error results and associated parameter optimisation values summarised in Table V. The 'BT LOS' model definition in (5) follows the 3GPP LOS model form built on the product of 


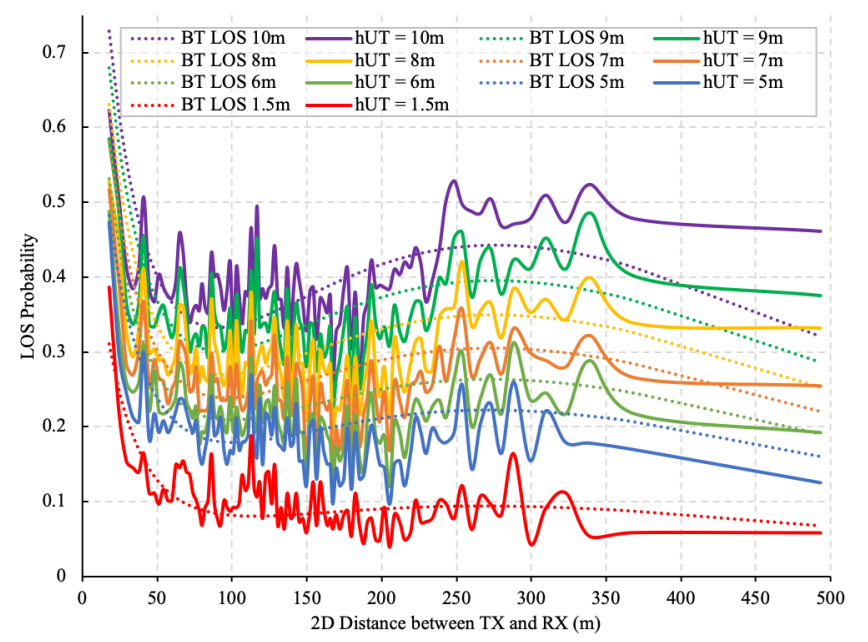

Fig. 9: Urban height dependent LOS probability.

two probability components but with modified parametrisation and linear scaling variables $C^{\prime\left(h_{U T}\right)}$ and $D^{\prime\left(h_{U T}\right)}$ in each component to account for end point height variation. Analysis is currently confined to, and validated for, endpoint heights up to $10 \mathrm{~m}$ as this is the antenna height assumed by 3GPP for urban microcellular (UMi) deployments. The proposed model shows good agreement for all $h_{U T}$ up to $10 \mathrm{~m}$ albeit with reduction in accuracy for cell edge probabilities as endpoint height increases.

Analysis of the DSM dataset crucially highlight distinct characteristics of increased probability and a local maximum toward the cell mid-point for endpoint heights $5 \mathrm{~m}$ and above. This is a property evident in all cell classification types resulting in the probability distribution functions becoming more clearly bi-modal rather than the assumed negative exponential form in current models. This characteristic is attributed to the point at which the endpoint height approaches that of the building height profile of the surrounding environment as building heights reduce with proximity to the cell centre. The clutter properties including the building height distribution profiles in the equivalent areas covered by the DSM have previously been analysed in [17].

$$
\begin{gathered}
\operatorname{Pr}_{L O S}=\left[\frac{D^{\prime}\left(h_{U T}\right)}{d_{2 D}}+\exp \left(-\frac{d_{2 D}}{a_{1}}\right)\left(1-\frac{D^{\prime}\left(h_{U T}\right)}{d_{2 D}}\right)\right] \\
\times\left(0.4+C^{\prime}\left(h_{U T}\right) \frac{5}{4}\left(\frac{d_{2 D}}{a_{2}}\right)^{3} \exp \left(-\frac{d_{2 D}}{a_{3}}\right)\right) \\
\text { Where } \\
C^{\prime}\left(h_{U T}\right)=\left(c_{1} h_{U T}\right)+c_{2} \\
D^{\prime}\left(h_{U T}\right)=\left(d_{1} h_{U T}\right)+d_{2}
\end{gathered}
$$

\section{CONCLUSIONS}

In this paper we evaluated the LOS statistical channel model using a representative 3D environmental model of a mobile

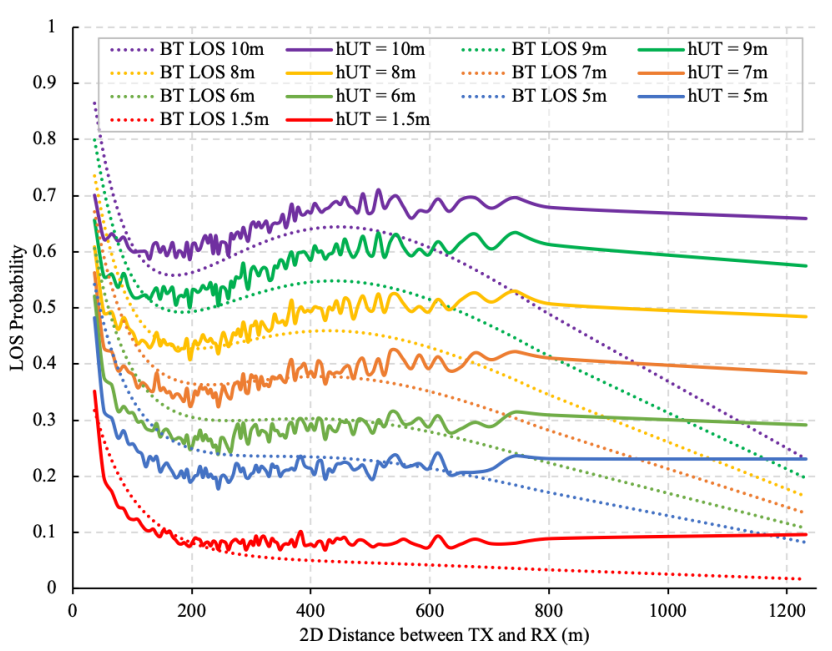

Fig. 10: Suburban height dependent LOS probability.

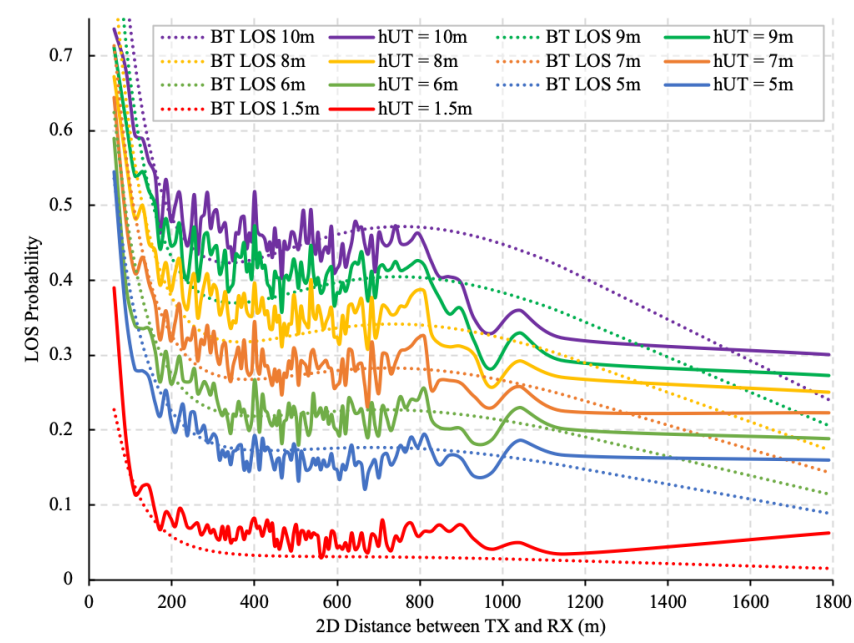

Fig. 11: Rural height dependent LOS probability.

network in the UK. Existing LOS probability models were assessed against the digital surface model using real lamp post and street lighting locations as representative outdoor data points distributed throughout the coverage environment of the network. Published models have been shown to be unsuitable for LOS predictions for all cell types when applied to a real network topology and geographic topography. As a result, the use of existing LOS probability models is insufficient for evaluation of use cases such as mmWave transport solutions between existing macro sites and new street infrastructure locations which may underpin future cell densification architectures. These findings are further supported with experimental verification of the methodology used, implying a revised statistical model suitable for such deployments is required. By extending the study to account for height dependency of the endpoint, a new model is proposed which includes height attributes of the endpoint below $10 \mathrm{~m}$. In addition, the newly proposed model integrates urban, suburban and 
TABLE V: Parametrisation of the BT LOS Probability Model.

\begin{tabular}{|c|c|c|c|}
\hline Model & Parameters & Height & MSE \\
\hline \multirow{7}{*}{ BT LOS (UMa) } & \multirow{7}{*}{$\begin{array}{c}a_{1}=20 \\
a_{2}=95 \\
a_{3}=150 \\
c_{1}=0.013 \\
c_{2}=0.38 \\
d_{1}=3.69 \\
d_{2}=5.47\end{array}$} & $h_{U T}=1.5 \mathrm{~m}$ & 0.07 \\
\hline & & $h_{U T}=5 \mathrm{~m}$ & 0.08 \\
\hline & & $h_{U T}=6 m$ & 0.09 \\
\hline & & $h_{U T}=7 m$ & 0.10 \\
\hline & & $h_{U T}=8 m$ & 0.13 \\
\hline & & $h_{U T}=9 \mathrm{~m}$ & 0.52 \\
\hline & & $h_{U T}=10 \mathrm{~m}$ & 0.19 \\
\hline \multirow{7}{*}{ BT LOS (SMa) } & \multirow{7}{*}{$\begin{array}{c}a_{1}=70 \\
a_{2}=192 \\
a_{3}=257 \\
c_{1}=0.039 \\
c_{2}=0.21 \\
d_{1}=14.26 \\
d_{2}=-3.49\end{array}$} & $h_{U T}=1.5 \mathrm{~m}$ & 0.03 \\
\hline & & $h_{U T}=5 m$ & 0.18 \\
\hline & & $h_{U T}=6 m$ & 0.17 \\
\hline & & $h_{U T}=7 m$ & 0.11 \\
\hline & & $h_{U T}=8 m$ & 0.08 \\
\hline & & $h_{U T}=9 m$ & 0.10 \\
\hline & & $h_{U T}=10 m$ & 0.12 \\
\hline \multirow{7}{*}{ BT LOS (RMa) } & \multirow{7}{*}{$\begin{array}{c}a_{1}=60 \\
a_{2}=235 \\
a_{3}=440 \\
c_{1}=0.01 \\
c_{2}=0.09 \\
d_{1}=20.86 \\
d_{2}=-12.21\end{array}$} & $h_{U T}=1.5 \mathrm{~m}$ & 0.07 \\
\hline & & $h_{U T}=5 m$ & 0.02 \\
\hline & & $h_{U T}=6 m$ & 0.04 \\
\hline & & $h_{U T}=7 \mathrm{~m}$ & 0.06 \\
\hline & & $h_{U T}=8 m$ & 0.11 \\
\hline & & $h_{U T}=9 \mathrm{~m}$ & 0.15 \\
\hline & & $h_{U T}=10 \mathrm{~m}$ & 0.15 \\
\hline
\end{tabular}

rural deployment scenarios into one common definition. The proposed 'BT LOS model' demonstrates good agreement for all scenarios allowing a wide range of use cases to be analysed at scale. The findings contribute significant insight into the fundamental propagation characteristics of real mobile networks including large scale parameter assignment for LOS and NLOS propagation conditions. Further work looking at the complimentary UMi LOS probability model is also under consideration as a means of assessing the potential transport network footprint achievable with application of street level mmWave multi-hop and mesh wireless x-haul solutions.

\section{REFERENCES}

[1] 3GPP, TR 21.915 V15.0.0 Technical Specification Group Services and System Aspects; Release 15 Description; Summary of Rel-15 Work Items, 2019.

[2] T. S. Rappaport, Y. Xing, G. R. MacCartney, A. F. Molisch, E. Mellios, and J. Zhang, "Overview of Millimeter Wave Communications for Fifth-Generation (5G) Wireless Networks - With a Focus on Propagation Models," IEEE Transactions on Antennas and Propagation, vol. 65, no. 12, pp. 6213-6230, 2017. DOI: 10.1109/TAP.2017.2734243.

[3] T. S. Rappaport, S. Sun, R. Mayzus, H. Zhao, Y. Azar, K. Wang, G. N. Wong, J. K. Schulz, M. Samimi, and F. Gutierrez, "Millimeter wave mobile communications for 5G cellular: It will work!" IEEE Access, vol. 1, pp. 335-349, 2013.

[4] mmMAGIC. (2017). Measurement Results and Final mmMAGIC Channel Models, H2020-ICT-671650mmMAGIC/D2.2, [Online]. Available: https : / / $5 \mathrm{~g}$ mmmagic.eu/results/.
[5] T. S. Rappaport, G. R. MacCartney, S. Sun, H. Yan, and S. Deng, "Small-scale, local area, and transitional millimeter wave propagation for $5 \mathrm{~g}$ communications," IEEE Transactions on Antennas and Propagation, vol. 65, no. 12, pp. 6474-6490, 2017.

[6] S. Rajagopal, S. Abu-Surra, and M. Malmirchegini, "Channel feasibility for outdoor non-line-of-sight mmwave mobile communication," in 2012 IEEE Vehicular Technology Conference (VTC Fall), 2012, pp. 1-6.

[7] 3GPP, TR 38.874 v16.0.0 Study on Integrated Access and Backhaul, 2019.

[8] NGMN, 5G RAN CU - DU network architecture, transport options and dimensioning, 2019.

[9] G. Interdonato, E. Björnson, H. Ngo, P. Frenger, and E. Larsson, "Ubiquitous cell-free massive mimo communications," EURASIP Journal on Wireless Communications and Networking, Aug. 2019. DOI: 10.1186/ s13638-019-1507-0.

[10] 3GPP, TR 38.900 V14.3.0 Study on channel model for frequency spectrum above $6 \mathrm{GHz}, 2017$.

[11] 5GCM. (2016). 5G Channel Model for bands up to 100 $\mathrm{GHz}$, [Online]. Available: http://www.5gworkshops . com/5GCM.html.

[12] M. K. Samimi, T. S. Rappaport, and G. R. MacCartney, "Probabilistic omnidirectional path loss models for millimeter-wave outdoor communications," IEEE Wireless Communications Letters, vol. 4, no. 4, pp. 357-360, 2015.

[13] ITU-R, M.2135-1 guidelines for evaluation of radio interface technologies for IMT-Advanced, 2009.

[14] WINNERII, WINNER II Channel Models, D1.1.2 V1.2, IST-4-027756 WINNER II Deliverable, 2008.

[15] 3GPP, TR 36.873 V2.0.0 Study on 3D-Channel Model for LTE, 2014.

[16] S.-E. Ericsson, Height Dependent LOS Probability for 3D-Channel Model, 2013.

[17] D. Townend and S. D. Walker, "A 3D statistical framework for the UK's mobile network," in 2020 IEEE 31st Annual International Symposium on Personal, Indoor and Mobile Radio Communications, Aug. 2020.

[18] Department for Environment, Food and Rural Affairs. (2019). Defra Data Services Platform Surveys, [Online]. Available: https://environment.data.gov.uk.

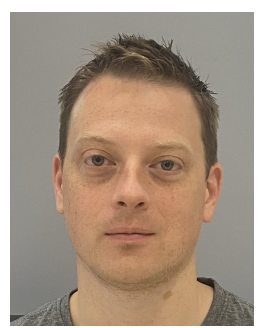

Dave Townend received the M.Eng degree in wireless communication engineering from Loughborough University, U.K., in 2007 and is currently a Wireless Research Manager at BT Laboratories. He is currently pursuing a Ph.D. degree with the School of Computer Science and Electronic Engineering at the University of Essex, U.K., and is a Chartered Engineer and a Member of the IET. His research interests include high frequency propagation, mobile network deployment modelling and wireless backhaul. 


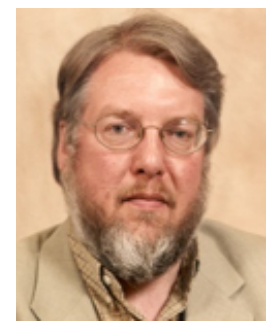

Stuart D. Walker received the B.Sc. (Hons) degree in physics from Manchester University, U.K., in 1973, the M.Sc. degree in telecommunications systems and $\mathrm{Ph} . \mathrm{D}$. degree in electronics from Essex University, Colchester, U.K., in 1975 and 1981 respectively. From 1981-82, he was a post-doctoral research assistant at Essex University. From 198287 , he was a research scientist at BT Laboratories, and from 1987-88 he was promoted to Group Leader in Submarine Systems Design. He joined Essex University in 1988 as a Senior Lecturer, and was promoted to Reader in 2002 and to Full Professor in 2004. At Essex University, he manages a laboratory concerned with all aspects of Access Networks: The Access Networks Group (ANG).

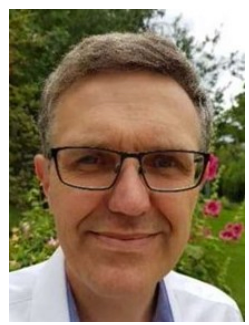

Adrian Sharples is the Senior Wireless Research Manager at BT Laboratories, leading a team of researchers to investigate the opportunities for applying new wireless techniques to mobile and fixed communications. He has over 30 years experience working on telecommunications access technologies in both technical and strategy roles. Adrian has a Ph.D. in telecommunications and is a Visiting Professor at the University of Kent., U.K. He is a Chartered Engineer and a Member of the IET.

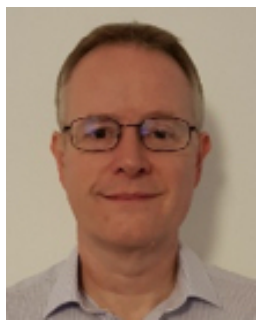

Andy Sutton is a Principal Network Architect in BT's Architecture and Technology Strategy team where he is responsible for radio access network (RAN) architecture evolution and mobile xhaul strategy. Andy holds an M.Sc. in mobile communications from the University of Salford and has 35 years of experience within the telecommunications industry. His research interests include distributed and centralised RAN architectures, optical and wireless transmission systems and mission critical network design and optimisation. Andy holds the post of Visiting Professor at the University of Liverpool and University of Salford, he's a Chartered Engineer and holds Fellowships from the IET, ITP and BCS. Andy sits on the editorial board of the Institute of Telecommunications Professionals Journal and has co-authored four books on telecommunications topics. 